\title{
Consideraciones Actuales en el Estudio Imagenológico de las Calcificaciones de Tejidos Blandos en Zona de Ángulo Mandibular
}

\author{
Currrent Considerations in the Study of Image of Soft \\ Tissue Calcification in Mandibular Angle Area
}

\author{
Ivonne Garay ${ }^{*}$ Sergio Olate ${ }^{* *}$
}

GARAY, I. \& OLATE, S. Consideraciones actuales en el estudio imagenológico de las calcificaciones de tejidos blandos en zona de ángulo mandibular. Int. J. Odontostomat., 7(3):455-464, 2013.

RESUMEN: Las calcificaciones de tejidos blandos en la región máxilo-facial son poco frecuentes, sin embargo, pueden representar hallazgos radiográficos en radiografías dentales de rutina, tales como Radiografías Panorámicas y Radiografías Cefalométricas Laterales. Estas calcificaciones se presentan como radiopacidades principalmente en la zona del ángulo mandibular, las que muchas veces pueden presentar dificultades en cuanto a su identificación y diagnóstico. Las calcificaciones pueden ser distróficas, idiopáticas o metastásicas, siendo estás últimas extremadamente infrecuentes en la región oral. Las calcificaciones mayormente descritas en la literatura son calcificaciones de arteria carótida, sialolitos, calcificaciones de los nodos linfáticos, tonsilolitos y flebolitos. La literatura disponible, sin embargo, es limitada en cuanto a cantidad y al valor de la evidencia; la mayoría de los estudios encontrados corresponden a reporte de casos. La siguiente revisión bibliográfica tiene como objetivo recolectar la información científica disponible con respecto a las calcificaciones distróficas y calcificaciones idiopáticas observables en ángulo mandibular, abordando los aspectos clínico e imagenológico.

PALABRAS CLAVE: calcinosis, radiografías panorámicas, calcificación.

\section{INTRODUCCIÓN}

La calcificación es un fenómeno bioquímico caracterizado por el depósito de sales de calcio normalmente presentes en tejidos óseos y dentarios. Aunque los niveles de calcio en el líquido extracelular son mínimos, su concentración es crítica para varias funciones y permanece constante, siendo regulada principalmente por la hormona paratiroidea. El fósforo, por otra parte, es un componente fundamental del hueso y es el principal anión de los fluidos intracelulares, cumpliendo funciones muy importantes en diferentes procesos enzimáticos celulares (Jacome \& Abdo, 2010).

Las calcificaciones patológicas conocidas como heterotópicas, se producen debido a los cambios del metabolismo celular que causa el depósito de sales anormales de calcio y otras sales en lugares donde no se depositan comúnmente (Freitas et al., 2004).
Según Freitas et al. y White \& Pharoah (2002), las calcificaciones patológicas pueden ser clasificadas en: (1) distróficas: este tipo de calcificación se produce ante niveles normales de calcio en la sangre, cuando no hay suficiente suministro de sangre y hay presencia de tejido isquémico y necrótico, dentro de este grupo se encuentran nodos linfáticos calcificados, tonsilolitos y calcificaciones de arteria carótida; (2) idiopáticas: Son depósitos de sales de calcio en tejidos blandos en presencia de niveles normales de calcio en la sangre; su origen es desconocido, a este tipo de calcificaciones se vinculan sialolitos y flebolitos; (3) metastásicas: Corresponden al precipitado mineral dentro del tejido blando como resultado de un mayor nivel sérico de calcio y fosfato (casos de hipervitaminosis D, hiperparatiroidismo e insuficiencia renal crónica). Este

* Programa de Magister en Ciencias Odontologicas, Universidad de La Frontera; Docente Unidad de Imagenología Oral y Maxilofacial, Universidad de La Frontera, Chile.

** Profesor Asistente Unidad de Cirugía Oral y Maxilofacial, Universidad de La Frontera, Chile.

*** Centro de Investigación en Ciencias Biomédicas. Universidad Autónoma de Chile, Chile. 
tipo de calcificaciones son extremadamente infrecuentes en la región oral. En el área odontológica las calcificaciones de tejidos blandos son generalmente hallazgos radiográficos, en radiografías panorámicas y Telerradiografías laterales (Jacome \& Abdo).

Las calcificaciones de tejido blando son infrecuentes, pudiendo encontrarse en el $4 \%$ de las Radiografías Panorámicas (Monsour et al., 1991). Los criterios más importantes a considerar en la interpretación radiográfica son la localización anatómica, la distribución, el número, el tamaño y la forma de las calcificaciones, siendo la anatomía regional un elemento fundamental en el diagnóstico (White \& Pharoah, 2002). El diagnóstico diferencial es realizado con el hueso hioide, epiglotis, cartílago tritíceo, proceso estiloides, asta superior del cartílago tiroides. Existen pocas referencias en la literatura sobre las técnicas imagenológicas para la identificación de las calcificaciones de tejido blando, gran parte de las publicaciones corresponden a reporte de casos clínicos (Jacome \& Abdo).

El objetivo de la presente revisión bibliográfica es analizar la literatura existente con respecto a las calcificaciones distróficas e idiopáticas que pueden ser encontradas en al área del ángulo mandibular, en exámenes radiográficos dentales de rutina, enfatizando aspectos clínicos e imagenológicos, con el fin de dar una orientación diagnóstica al cirujano-dentista (Tabla I).

\section{Calcificaciones Distróficas}

Nodos linfáticos calcificados. La calcificación de nodos linfáticos es poco frecuente y afecta a nodos con inflamación crónica. Generalmente son asintomáticos y se presentan como masas duras redondas o elongadas de ser palpables. Los nodos más afectados son los sub-mandibulares y los cervicales. El diagnóstico diferencial debe realizarse con sialolito en la región del hilio de glándula sub-mandibular; los nodos calcificados tienen un contorno irregular y aspecto interno indefinido, pudiendo variar el grado de radiopacidad, exibiendo un aspecto laminar o lobular. (White \& Pharoah, 2002; Monsour et al., 1991) (Fig.1).

No existen estudios en donde se evalúe únicamente presencia de nodos calcificados mediante radiografías dentales. Las pocas investigaciones realizadas con respecto a nodos cervicales calcificados tratan del diagnóstico mediante Tomografía computarizada y Ultrasonografía.

Eisenkraft et al. (1999) estudiaron si la presencia y el patrón de calcificación de los nodos linfáticos pueden ser utilizados para hacer un diagnóstico diferencial entre una enfermedad benigna o maligna. Se analizaron 2.300 Tomografías Computarizadas de área cervical, $26(1 \%)$ presentaban calcificación de nodos linfáticos. De estos 26 individuos, $8(31 \%)$ eran pacientes con enfermedad benigna, $6(23 \%)$ correspondían a pacientes con enfermedades tratadas, incluyendo cua-

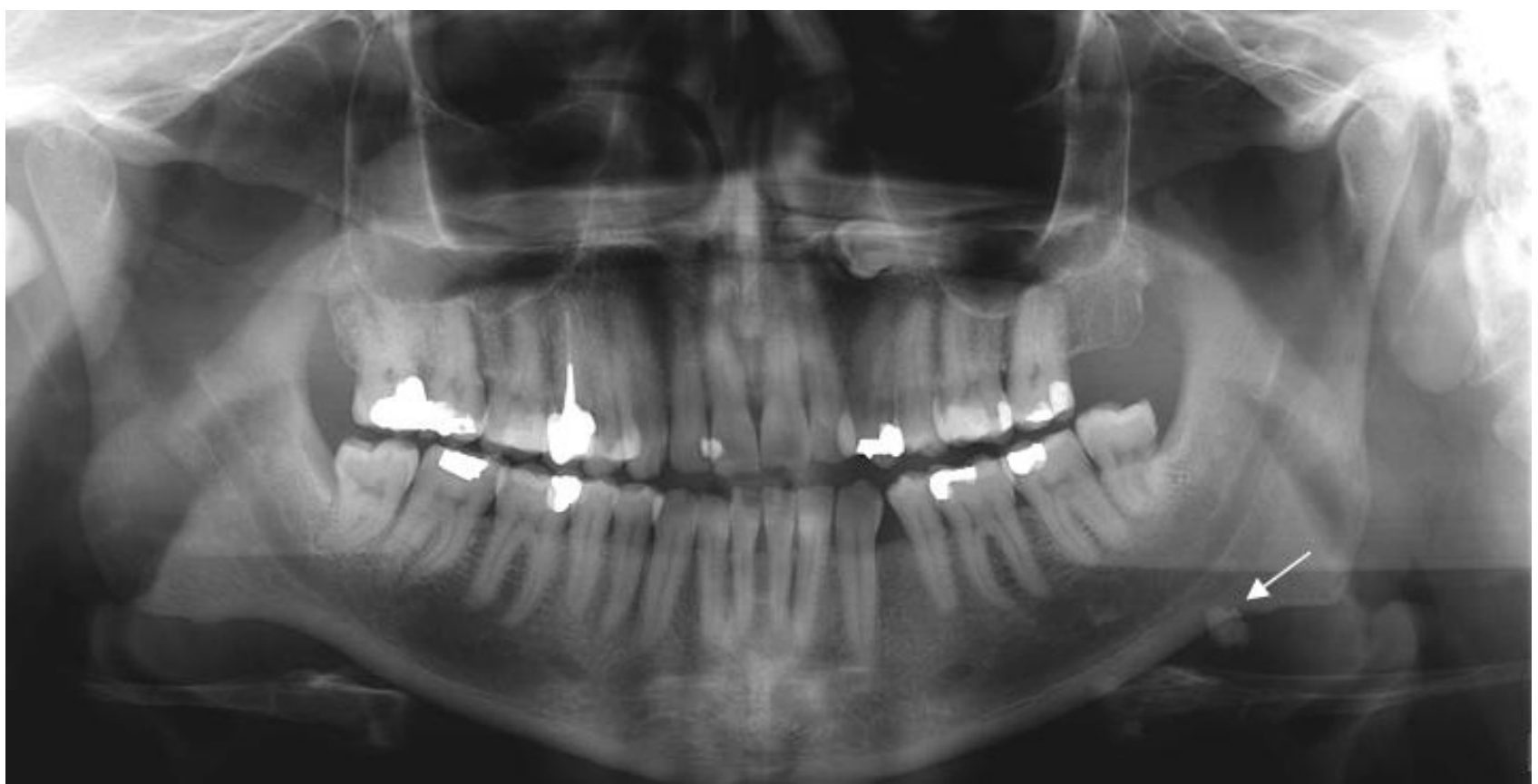

Fig. 1. Nodo linfático calcificado, sobreproyectado en margen mandibular inferior de lado izquierdo. 
GARAY, I. \& OLATE, S. Consideraciones actuales en el estudio imagenológico de las calcificaciones de tejidos blandos en zona de ángulo mandibular. Int. J. Odontostomat., 7(3):455-464, 2013.

Tabla I. Estudios imagenológicos de calcificaciones de tejidos blandos en zona de ángulo mandibular.

\begin{tabular}{|c|c|c|c|c|}
\hline Autor & Calcificaciones & Diseño de estudio & $\begin{array}{c}n \text { de } \\
\text { individuos }\end{array}$ & Estudio imagenológico \\
\hline Monsour et al. (1991) & Calcificaciones en general & Corte transversal & 2628 & $\mathrm{RP}$ \\
\hline Jacome \& Abdo (2010) & Calcificaciones en general & Revisión bibliográfica & -- & $\mathrm{RD}$ \\
\hline Eis enkraft et al. (1999) & Nódulos calcificados & Corte transversal & 2300 & TC \\
\hline Choi et al. (2009) & Nódulos calcificados & Reporte de casos & 2 & US \\
\hline Cogolludo et al. (2002) & Tonsilditos & Reporte de casos & 1 & ELC, TC \\
\hline Ram et al. (2004) & Tonsilditos & Reporte de casos & 1 & $\mathrm{RP}$ \\
\hline Silvestre et al. (2005) & Tonsilditos & Reporte de casos & 1 & $\mathrm{TC}$ \\
\hline De Moura et al. (2007) & Tonsilditos & Reporte de casos & 3 & $\mathrm{RP}$ \\
\hline Thakur et al. (2008) & Tonsilditos & Reporte de casos & 1 & TC \\
\hline Kamikawa et al. (2006) & Calcifica ción de arteria carótida & Revisión bibliográfica & --- & $\mathrm{RP}$ \\
\hline Friedlander \& Lande (1981) & Calcifica ción de arteria carótida & Corte transversal & 182 & $\mathrm{RP}$ \\
\hline Friedlander \& Baker (1994) & Calcificación de arteria carótida & Corte transversal & 295 & $\mathrm{RP}$ \\
\hline Friedlander et al. (1994) & Calcificación de arteria carótida & Corte transversal & 19 & $\mathrm{RP}, \mathrm{US}$ \\
\hline Friedlander \& Gratt (1994) & Calcifica ción de arteria carótida & Corte transversal & 134 & $\mathrm{RP}$ \\
\hline Carter et al. (1997) & Calcifica ción de arteria carótida & Corte transversal & 1175 & $\mathrm{RP}$ \\
\hline Almog et al. (2004) & Calcifica ción de arteria carótida & Revisión bibliográfica & --- & $\mathrm{RP}$ \\
\hline Mupparapu et al. (2007) & Calcificación de arteria carótida & Revisión sistemática & --- & $\mathrm{RP}$ \\
\hline Tanaka et al. (2006) & Calcifica ción de arteria carótida & Cohorte & 659 & $\mathrm{RP}$ \\
\hline Griniatsos et al. (2009) & Calcifica ción de arteria carótida & Casos y controles & 40 & RP \\
\hline Romano-Sousa et al. (2009) & Calcifica ción de arteria carótida & Pruebas diagnósticas & 16 & $\mathrm{RP}, \mathrm{US}$ \\
\hline Rebolledo et al. (2009) & Sialolitos & Revisión bibliográfica & --- & $\mathrm{RD}$ \\
\hline El Deeb et al. (1981) & Sialolitos & Revisión bibliográfica & --- & $\mathrm{RD}$ \\
\hline Scolozzi et al. (2003) & Flebolitos & Reporte de casos & 1 & $\mathrm{RD}$ \\
\hline
\end{tabular}

RP: Radiografía Panorámica, TC: Tomografía Computarizada, US: Ultrasonografía, RD: Radiografía Dental, RLC: Radiografía Lateral de Cráneo.

tro linfomas, una histiocitosis y una infección aguda no especificada en el estudio. Doce de los pacientes (46\%) tenían enfermedades malignas dentro de las cuales se incluían tumores de cabeza y cuello y metástasis de adenocarcinoma de tumores de mama y de pulmón primario. La investigación concluye que la calcificación es poco frecuente y que, basándose en el limitado número de casos encontrados, la presencia de estas calcificaciones no sería un predictor fiable de que la enfermedad sea benigna o maligna. Sin embargo, la calcificación nodular linfática sugiere un diagnóstico diferencial más limitado que incluye a la tuberculosis, linfoma tratado, carcinoma metastásico de tiroides, adenocarcinoma y carcinoma epidermoide.

Choi et al. (2009) reportaron dos casos clínicos en pacientes con carcinoma papilar de tiroides los cuales presentaron nodos cervicales con necrosis quística y calcificación sugerente de metástasis, diagnosticada previamente con ultrasonografía. Sin embargo, después de la disección de cuello y resección de tumor tiroideo se observó persistencia de linfadenopatía. En el primer caso, una mujer de 49 años, el examen histopatológico pos quirúrgico reveló que la linfadenopatía cervical se debía a tuberculosis. El segundo caso, una mujer de 52 años, había padecido tuberculosis pulmonar treinta años atrás sin antecedentes de recidiva, finalmente el nodo linfático anormal correspondía a una linfadenitis tuberculosa.

Tonsilolitos. Los tonsilolitos son calcificaciones distróficas poco frecuentes, causadas por inflamación crónica de las tonsilas palatinas. Estas calcificaciones pueden ser observadas en individuos de ambos sexos, en un amplio rango etáreo, siendo la mayoría de los casos reportados en adultos mayores. Los tonsilolitos se forman a partir del material retenido, crecimiento de bacterias y hongos en las criptas. En su composición química se encuentran principalmente sales de calcio, en especial hidroxiapatita y apatita de carbonato de calcio (Cogolludo et al., 2002). 
En radiografías panorámicas generalmente se observan formaciones pequeñas y múltiples sobreproyectadas en la parte media de la rama mandibular, de contornos mal definidos y de densidad ligeramente más radiopaca que el hueso esponjoso. Se debe considerar en el diagnóstico diferencial masas radiopacas observables en la rama mandibular, como islotes aislados de hueso denso (White \& Pharoah, 2002) (Fig. 2).

Los tonsilolitos generalmente son asintomáticos, sin embargo, clínicamente pueden presentarse como cuadros de halitosis y odinofagia, acompañados de sensación de cuerpo extraño y otalgia refleja. En la exploración se aprecian como estructuras duras y blanquecinas que ocupan las criptas, no obstante, la exploración de la tonsila puede no ser llamativa. El diagnóstico diferencial debe hacerse con tonsilitis agudas y crónicas, hipertrofia tonsilar, abscesos peri-tonsilares, cuerpos extraños, flebolitos, tejidos ectópicos de hueso o cartílago, nódulos linfáticos y enfermedades granulomatosas. Deben ser considerados además pacientes con osificación del ligamento estilohioideo o proceso estiloide elongado que pueden presentar sintomatología similar en presencia de Síndrome de Eagle (Cogolludo et al., 2002; Silvestre et al., 2005).

Ram et al. (2004) reportaron un caso de tonsilolito unilateral que simulaba ser bilateral en una radiografía panorámica. El caso correspondía a una mujer de la India de 57 años. Los autores hacen referencia a que la superposición de una lesión en un lado de la mandíbula puede crear una imagen fantasma en el lado contrario lo que puede llevar a un mal diagnóstico de lesiones bilaterales. Por esta razón, el artículo concluye que otros estudios de imagen como una tomografía computarizada o una resonancia magnética pueden ser necesarias para distinguir las imágenes fantasmas de una verdadera patología bilateral.

Silvestre et al. (2005) presentan en su artículo el caso de una mujer de 55 años de edad que tenía sintomatología de disfagia y molestias en la faringe con sensación de cuerpo extraño. Al examen clínico se palpaba una tumoración de consistencia dura a nivel submucoso en el paladar blando, a nivel del pilar tonsilar anterior derecho, con leve eritema de la mucosa. El estudio imagenológico utilizado para realizar el diagnóstico fue tomografía computarizada, en la cual se mostraba una imagen ovalada delimitada y calcificada de gran tamaño $(2,5 \times 1,5 \mathrm{~cm})$. El tratamiento realizado fue exéresis quirúrgica de dicha estructura.

De Moura et al. (2007) relataron tres casos asintomáticos de tonsilolitos, descubiertos mediante radiografías panorámicas de rutina. Un caso correspondía a un hombre de 65 años, evaluado para colocación de implantes, cuya radiografía panorámica mostró múltiples áreas radiopacas bilaterales sobreproyectadas en rama mandibular y en el cartíla-

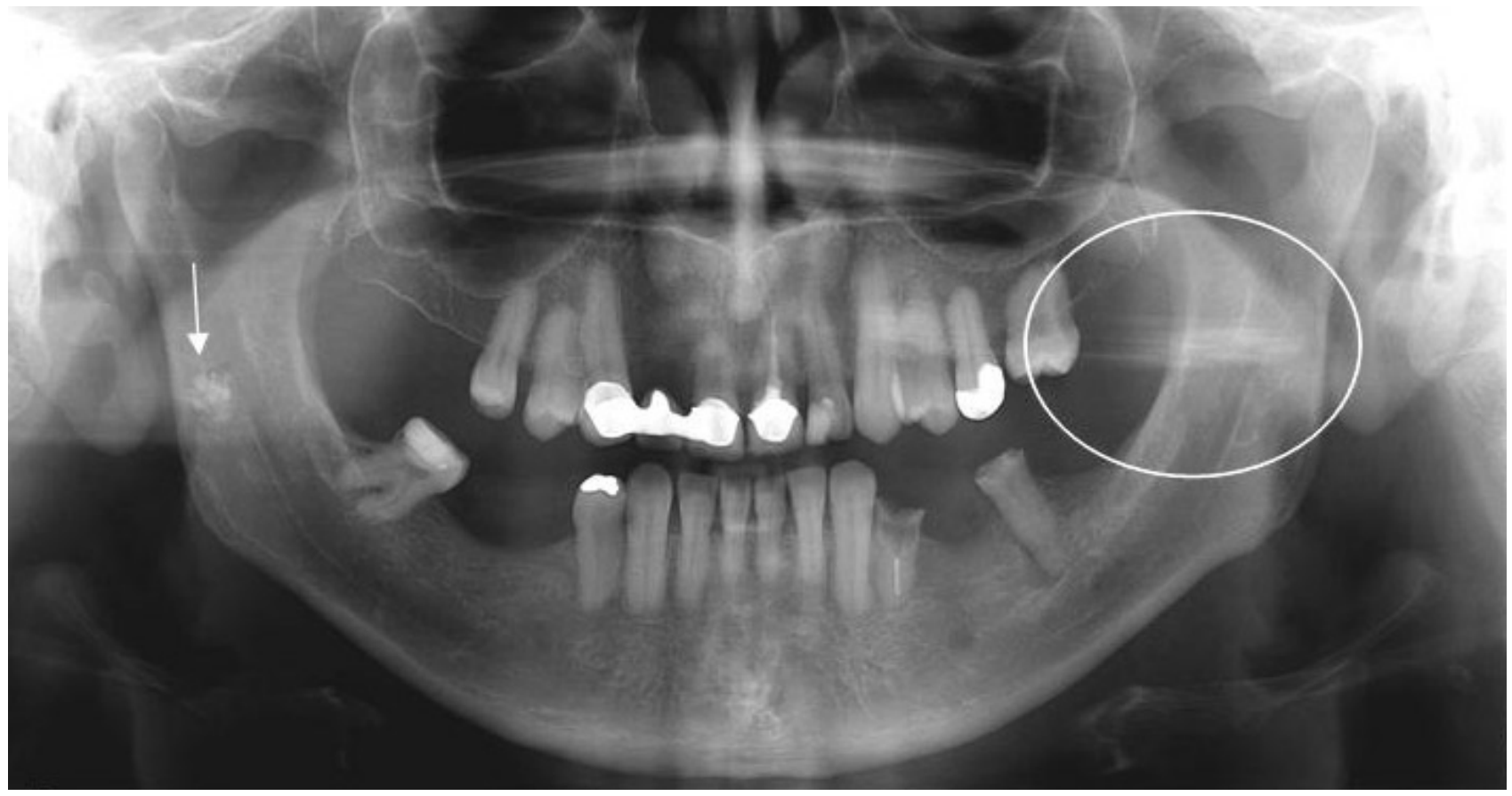

Fig. 2. Tonsilolitos sobreproyectados en rama mandibular derecha, imagen fantasma en rama mandibular izquierda. 
go de la oreja, con un diámetro promedio de $5 \mathrm{~mm}$. El segundo caso correspondía a una mujer de 71 años, evaluada para colocación de implantes, la que presentó imágenes radiopacas de $3 \mathrm{~mm}$ de diámetro, sobreproyectadas en la rama mandibular derecha. El último caso correspondía a un hombre de 42 años en seguimiento por un ameloblastoma recurrente y cuyo examen radiográfico mostró imágenes radiopacas bilaterales de $2 \mathrm{~mm}$ de diámetro sobreproyectadas en rama mandibular y en paladar blando. No se encontraron síntomas en ninguno de los casos presentados. Sólo el caso uno tenía características clínicas, en el que se observó presencia de placas blancas, visibles a través de la mucosa. Para localizar exactamente la ubicación de estas imágenes, se solicitó Tomografía Computarizada. Los autores concluyen que los tonsilolitos pueden mostrar imágenes en las radiografías panorámicas similares a alteraciones intra-óseas y que el diagnóstico es relativamente fácil cuando se realiza una tomografía computarizada.

Aunque la mayoría de los casos reportados se refieren a pacientes adultos. Thakur et al. (2008) reportaron el caso de una niña con dolor leve a moderado y no irradiado durante la deglución con un año de evolución. No se observaron otros síntomas asociados y al examen clínico se observó mayor tamaño de las tonsilas y grandes áreas blanco tiza en la tonsila izquierda. Mediante Tomografía Computarizada, se detectó una gran área radiopaca de 3,9 × 3,4 cm en la zona de la tonsila, diagnosticado como tonsilolito gigante. Se realizó tonsilectomía bajo anestesia general y se extrajo la calcificación.

\section{Calcificación de arteria carótida}

Las placas de ateromas se forman dentro de las arterias debido a la deposición de colesterol en la íntima debido a daño del endotelio, causado por factores de riesgo tales como la hipertensión, derivados del hábito de fumar, el colesterol alto, obesidad, etc. Se produce una respuesta inflamatoria debido a la proliferación de fibroblastos causando engrosamiento de la íntima y el endurecimiento arterial. Se inicia, entonces la deposición de sales de calcio, que produce diferentes grados de calcificación distrófica. Las arterias afectadas son la aorta, coronarias y cerebrales, incluyendo la arteria carótida (Kamikawa et al., 2006).

Al analizar radiografías panorámicas el diagnóstico diferencial de calcificaciones de la arteria carótida se realiza con radiopacidades anatómicas tales como el hueso hioides, la epiglotis, el cartílago tritíceo, cuerno superior del cartílago tiroides, calcificación de los ligamentos estilohioideo y estilomandibular (Fig. 3). También se deben diferenciar de radiopacidades patológicas como nodos linfáticos calcificados, sialolitos, flebolitos y tonsilolitos (Kamikawa et al., 2006; White \& Pharoah; Friedlander et al. 1994a).

Las radiografías dentales se limitan únicamente a la identificación de la placa de ateroma, pero no permite la evaluación de su ubicación exacta y el grado de obliteración arterial, por lo que es necesario el uso de pruebas más específicas, como la ecografía y la tomografía computarizada (Friedlander et al., Kamikawa et al.).

Friedlander \& Lande (1981) comenzaron estudios referidos al diagnóstico de calcificaciones a nivel de la bifurcación, basándose en que $2 / 3$ de los accidentes cerebro vasculares (ACV) están causados por la formación de trombos y émbolos en la región de la bifurcación de la arteria carótida, postularon la posibilidad de identificar a través de la radiografías panorámicas las placas de ateroma calcificadas a nivel de esta bifurcación y que serían causantes de la formación de dichos émbolos. Estas calcificaciones podrían dar imágenes como masas nodulares radiopacas 0 como dos líneas verticales radiopacas adyacentes o justo debajo del espacio intervertebral entre C3 y C4, aproximadamente 1 a $2,5 \mathrm{~cm}$ inferior y posterior al ángulo de la mandíbula.

Friedlander et al., investigaron la presencia de lesiones carotídeas calcificadas en radiografías panorámicas de rutina en una población de 182 personas atendidas en un centro médico de veteranos del ejército, de 55 años o más, sin sintomatología o antecedentes cardiovasculares. Informaron que de los pacientes examinados, cinco hombres y una mujer $(3,3 \%)$, con una edad promedio de 67,7 años, presentaban calcificaciones en la arteria carótida.

Friedlander et al. (1994b) realizaron otro estudio de 295 personas de 55 años o más, asintomáticos y sin historia de accidente vascular, para determinar la prevalencia de lesiones ateroscleróticas calcificadas de la carótida en radiografías panorámicas previas al tratamiento. De estos pacientes nueve hombres y una mujer (3,4\%), con una edad promedio de 69,3 años, presentaban calcificaciones, posteriormente esto fue confirmado con ultrasonido.

Friedlander et al. (1994c) detectaron mediante 


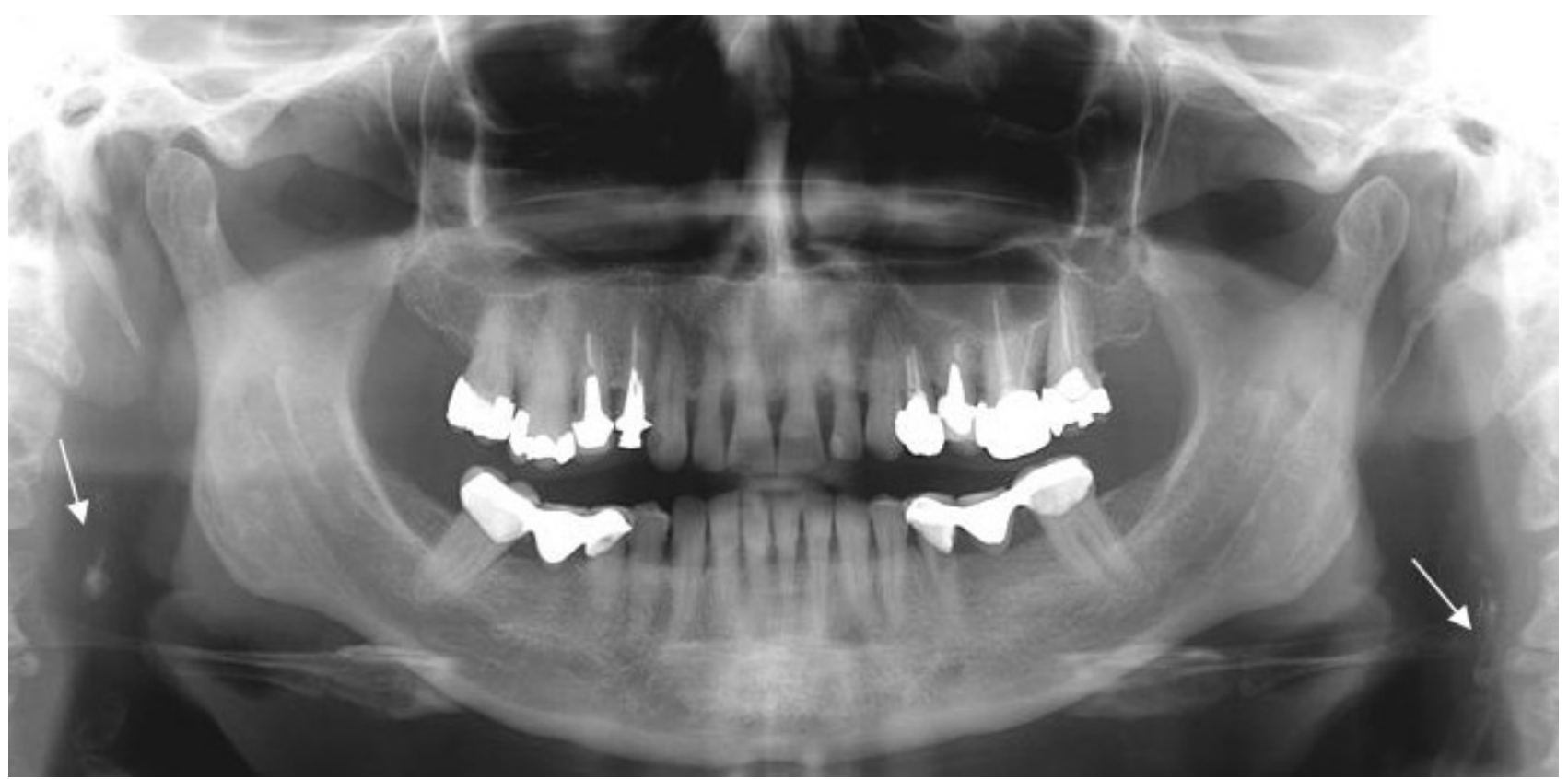

Fig. 3. Calcificaciones de arteria carótida bilaterales a nivel de $3^{a}$ vértebra cervical. Asta superior de cartílago tiroides y cartílago tritíceo observables a nivel de $4^{\text {a }}$ vértebra cervical.

radiografías panorámicas la presencia de calcificaciones en arteria carótida en $37 \%$ de los 19 hombres blancos admitidos en un centro médico de veteranos del ejército para tratamiento posterior a un ACV. Esto fue verificado por correlación con radiografías de la columna cervical, angiografía o Doppler. Carter et al. (1997) realizaron el primer estudio de prevalencia en la población ambulatoria general, encontraron calcificaciones vasculares carotídeas en $3,6 \%$ de radiografías panorámicas en una población ambulatoria de 1.175 pacientes. Los hombres eran $573(45,7 \%)$ de los pacientes y las mujeres eran 602 (54,3\%). La edad promedio fue de 40,1 años. Se evaluó la relación entre calcificaciones de arteria carótida con factores de riesgo para el desarrollo de ACV tales como hipertensión, obesidad, hipercolesterolemia, triglicéridos elevados, tabaquismo diabetes mellitus insulino-dependiente. Sólo la obesidad tuvo una asociación estadísticamente significativa.

Almog et al. (2004) revisaron los artículos publicados entre 1981 y 2003 en los que se informaba de la presencia de calcificaciones carotídeas detectables en las radiografías panorámicas. Estos estudios documentaron una prevalencia del $3 \%$ al $5 \%$ para la población general, siendo mayor para la población de riesgo.

En la revisión sitemática de Mupparapu et al. (2007), se analizaron 54 estudios que cumplían con la lista REMARK (Reporting Recommendations for Tumor Marker Prognostic Studies), en los que se encontró una asociación no significativa entre ateromas calcificados de arteria carótida y el riesgo de ACV entre la población estudiada, por lo tanto, la evidencia existente se consideró incompleta y no concluyente. Plantean que a menos que se realicen estudios de casos y controles o de cohortes que incluyan el control de los individuos que presenten ateromas en arteria carótida que conduzca a un ACV, la asociación entre los dos no tiene fundamento.

Dentro de los estudios analizados en esta revisión sistemática, el único estudio que cumplía la mayoría de los criterios de inclusión de REMARK fue el realizado por Tanaka et al. el año 2006. Ellos evaluaron si la presencia de ateromas en arteria carótida era un predictor de la enfermedad vascular en las radiografías panorámicas, mediante un estudio de cohorte. En el año 1998, se evaluaron 659 radiografías panorámicas de 262 hombres y 397 mujeres de 80 años de edad, sus datos generales y examen médico. Después de 5 años se evaluó la aparición de enfermedades vasculares en 191 sujetos y las causas de muerte en 108 sujetos que fallecieron en el período. No se encontró diferencia significativa en la ocurrencia de muerte vascular relacionada con la enfermedad en los sujetos con y sin calcificaciones en la arteria carótida. 
Griniatsos et al. (2009) realizaron un estudio en 40 pacientes con indicación de endarterectomía carotídea, a quienes se les tomó radiografía panorámica como parte del protocolo preoperatorio. Se compararon dos grupos, el de pacientes en los que se detectaron calcificaciones $(n=28)$ y el de los pacientes en quienes no se detectaron calcificaciones $(n=12)$ en la radiografía panorámica. El análisis estadístico entre los factores de riesgo para el desarrollo de accidente cerebrovascular entre los dos grupos de pacientes reveló una incidencia significativamente menor de diabetes mellitus, pero una mayor incidencia de placas de ateroma asociadas a sintomatología en el grupo de pacientes con calcificaciones detectables en la radiografía panorámica.

Romano-Sousa et al. (2009) investigaron la concordancia entre el diagnóstico de ateromas calcificados en las radiografías panorámicas y el Doppler color, considerado éste último como el estudio de referencia. Consideraron el hecho de que las imágenes panorámicas pueden mostrar la presencia de ateroma, independientemente del nivel de la obstrucción detectada por las imágenes Doppler. Se evaluaron ambos lados de cada paciente, con un total de 32 regiones cervicales analizadas. Se encontró un alto nivel de acuerdo, con un valor Kappa de 0,78. Los autores concluyen que las radiografías panorámicas pueden contribuir a la detección de calcificaciones en la región cervical de los pacientes susceptibles a las enfermedades vasculares que predisponen al infarto de miocardio y los accidentes cerebro vasculares.

\section{Calcificaciones Idiopáticas}

Sialolitos. La sialolitiasis es una entidad patológica que consiste en la obstrucción mecánica de la glándula salival y de su conducto excretor por formación de cálculos en el parénquima, secundariamente puede producirse infección de la misma, provocando un cuadro de sialolitiasis crónica. Estas formaciones calcáreas pueden ser únicas o múltiples y su forma puede variar entre ovalada y redondeada (Rebolledo et al., 2009). La sialolitiasis es la enfermedad más común de las glándulas salivales mayores después de la parotiditis y representa aproximadamente el $30 \%$ de todas alteraciones salivares, afecta a $0,01-1,0 \%$ de la población, con una mayor incidencia en los varones con edades comprendidas entre los 30 y 60 años (El Deeb et al., 1981).
La localización más común es en la glándula submandibular donde se encuentran el $92 \%$ de los cálculos (Fig. 4). Los cálculos generalmente consisten en una mezcla de diferentes fosfatos de calcio (principalmente hidroxi-apatita y carbonato-apatita), junto con una matriz orgánica. Los factores etiológicos implicados en la formación del sialolito se pueden deber a la retención de saliva debido a factores morfo-anatómicos, a factores de composición de la saliva y por la presencia de microorganismos, células epiteliales descamadas y cuerpos extraños (Grases et al., 2003).

Los sialolitos pueden observarse superpuestos en el ángulo y rama mandibular. Los cálculos posteriores pueden observarse en las radiografías lateral oblicua y panorámica. El diagnóstico diferencial incluye a los nodos linfáticos calcificados, en este caso los sialolitos se distinguen porque están asociados a dolor e hinchazón de la glándula submandibular (White \& Pharoah, 2002).

Aunque estas calcificaciones se presentan en individuos adultos, se han reportado casos en niños $\mathrm{y}$ adolescentes asociados a sialoadenitis aguda y crónica, secundaria a la inflamación asociada con esta patología. Zenk et al. (1999) realizaron un estudio en 635 pacientes, observaron sialolitiasis de la glándula submandibular con mayor frecuencia en pacientes entre 31 y 55 años de edad, y sólo el 6,1\% de los pacientes con esta patología eran menores de 20 años. Reuter et al. (1976) en una revisión que abarca un período de aproximadamente 100 años sólo encontraron 21 casos documentados de esta patología de la glándula submandibular en niños entre 3 semanas y 15 años de edad. Al igual que en los adultos, el principal síntoma es una hinchazón dolorosa de la glándula afectada que disminuye después de comer.

Ellies \& Laskawi (2010) reportaron 18 casos de niños, los que constituyen un grupo seleccionado de pacientes que se presentaron para tratamiento quirúrgico. El $66 \%$ de los sialolitos se encontraron en la porción proximal del conducto submandibular y el $33 \%$ fueron localizados en la porción distal del conducto excretor e hilio.

Flebolitos. Los flebolitos son calcificaciones idiopáticas de los trombos. En cabeza y el cuello están asociados con frecuencia a lesiones vasculares, que se clasifican en dos entidades clínicas: los hemangiomas y las malformaciones 


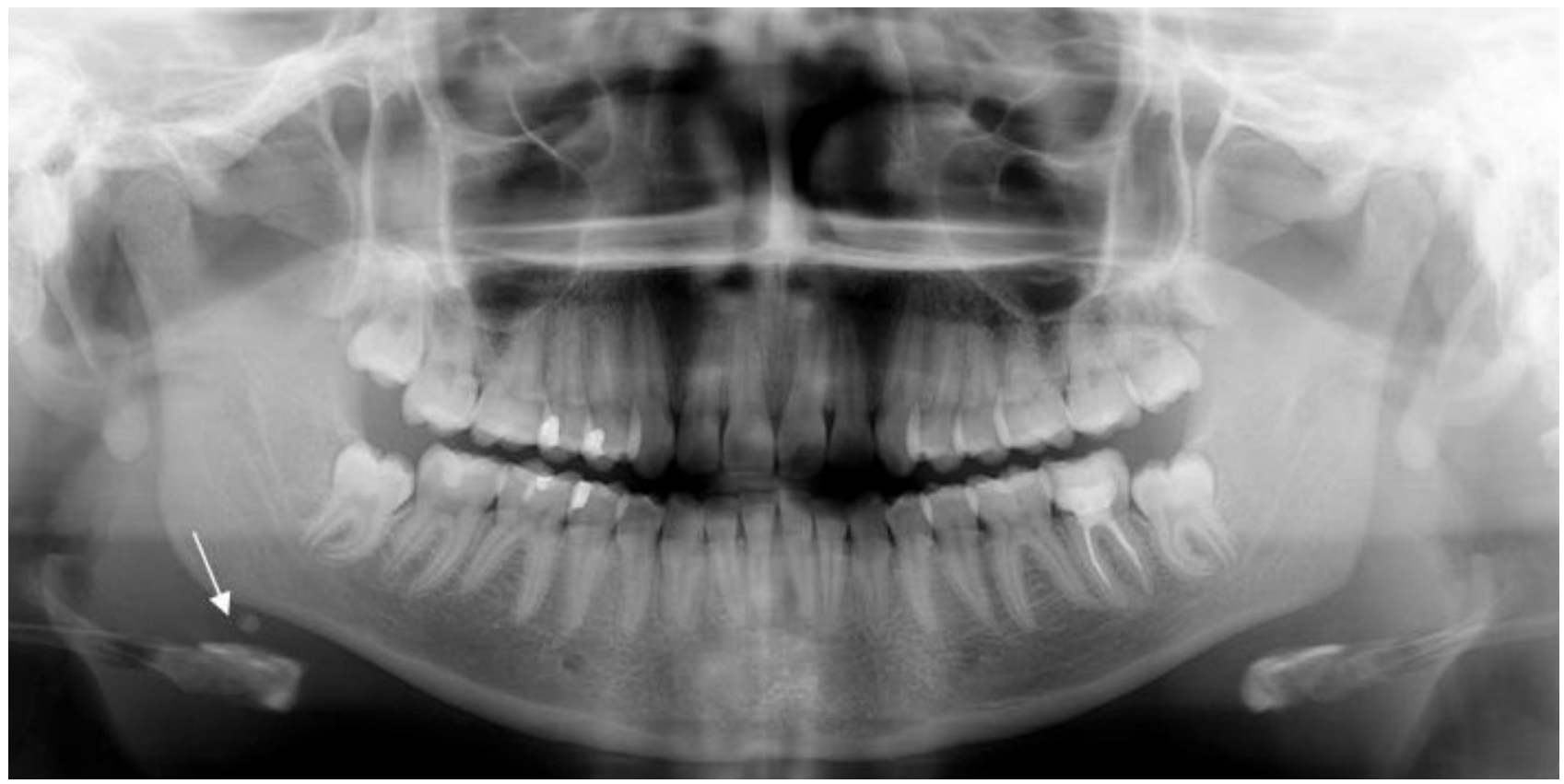

Fig. 4. Sialolito de glándula sub-mandibular derecha.

vasculares (Scolozzi et al., 2003). Estas calcificaciones pueden verse en radiografías panorámicas y postero-anteriores, presentándose como múltiples cuerpos laminados de forma circular u ovalada con un rasgo característico de halo radiopaco en la periferia y radiolúcido en centro, con aspecto radiopaco homogéneo interior y apariencia de láminas concéntricas.

Los flebolitos pueden tener una imagen similar a los sialolitos, sin embargo, éstos generalmente son únicos, de ser múltilples, éstos se orientan en una línea recta, en cambio los flebolitos son múltiples y se distribuyen al azar (White \& Pharoah). Es importante destacar que los hallazgos radiológicos de flebolitos en tejido blando de cabeza y el cuello constituyen evidencia de presencia de lesiones vasculares (Scolozzi et al., 2003).

\section{Consideraciones Finales}

Finalmente podemos señalar que las calcificaciones de tejidos blandos en la zona de ángulo mandibular son infrecuentes. La mayoría de ellas son asintomáticas y pueden ser hallazgos en radiografías panorámicas o cefalométricas laterales. Sin embargo, cuando estas calcificaciones tienen una implicancia clínica, su diagnóstico debe utilizar otras técnicas de imagen, tales como tomografía computarizada, ultrasonografía y resonancia magnética. La información disponible es de valor limitado en cuanto a la evidencia. Existe escasa información con respecto a algunas calcificaciones de tejido blando, en particular, de flebolitos, nodos calcificados y tonsilolitos. Por otro lado, no se observan aportes relevantes de los temas discutidos en esta revisión en los últimos veinte años.

GARAY, I. \& OLATE, S. Currrent considerations in the study of image of softtissue calcification in mandibular angle area. Int. J. Odontostomat., 7(3):455-464, 2013.

ABSTRACT: Soft tissue calcifications in the maxillofacial region are rare, however, may represent radiographic findings in routine dental radiographs such as panoramic radiographs and lateral cephalometric radiographs. These calcifications are presented as radiopacities mainly in the mandibular angle, sometimes in dentist may have difficulty in their identification and diagnosis. Calcification can be dystrophic, idiopathic or metastatic, the latter being extremely rare in the oral region. Calcifications largely described in the literature are carotid artery calcifications, sialoliths calcification of lymph nodes, tonsil stones and phleboliths. The available literature, however, is limited in quantity and value of the evidence, most of the studies found correspond to case reports. The following literature review aims to collect scientific information regarding idiopathic dystrophic calcifications and calcifications observed in mandibular angle, addressing clinical and imaging aspects.

KEY WORDS: calcinosis, panoramic radiograph, calcification. 


\section{REFERENCIAS BIBLIOGRÁFICAS}

Almog, D. M.; Illig, K. A.; Carter, L. C.; Friedlander, A. H.; Brooks, S. L. \& Grimes, R. M. Diagnosis of non-dental conditions. Carotid artery calcifications on panoramic radiographs identify patients at risk for stroke. $N$. Y. State Dent. J., 70(8):20-5, 2004.

Carter, L.; Haller, A.; Nadarajah, V.; Calamel, A. \& Aguirre, A. Use of panoramic radiography among an ambulatory dental population to detect patients at risk of stroke. $J$. Am. Dent. Assoc., 128(7):977-84, 1997.

Cogolludo, F.; Martín, G.; Olalla, A. \& Poch, J. A propósito de un caso: gran tonsilolito en amígdala palatina. Acta Otorrinolaringol. Esp., 53(3):207-10, 2002.

Choi, E.; Moon, W. \& Lim, Y. Tuberculous cervical lymphadenitis mimicking metastatic lymphnodes from papillary thyroid carcinoma. Case report. Br. J. Radiol., 82(982):208-11, 2009.

de Moura, M.; Madureira, D.; Noman-Ferreira, L.; Abdo, E.; de Aguiar, E. \& Freire, A. Tonsillolith: A report of three clinical cases. Med. Oral Patol. Oral Cir. Bucal, 12(2):E130-3, 2007.

Eisenkraft, B. L. \& Som, P. M. The spectrum of benign and malignant etiologies of cervical node calcification. AJR Am. J. Roentgenol., 172(5):1433-7, 1999.

El Deeb, M.; Holte, N. \& Gorlin, R. J. Submandibular salivary gland sialoliths perforated through the oral floor. Oral Surg. Oral Med. Oral Pathol., 51(2):134-9, 1981.

Ellies, M. \& Laskawi, R. Diseases of the salivary glands in infants and adolescents. Head Face Med., 6:1, 2010.

Freitas, A.; Rosa, J. \& Souza, I. Radiología Odontológica. $6^{\text {a }}$ ed. Sao Paulo, Artes Médicas, 2004.

Friedlander, A. H. \& Baker, J. D. Panoramic radiography: an aid in detecting patients at risk of cerebrovascular accident. J. Am. Dent. Assoc., 125(12):1598-603, 1994.

Friedlander, A. H. \& Gratt, B. M. Panoramic dental radiography as an aid in detecting patients at risk for stroke. J. Oral Maxillofac. Surg., 52(12):1257-62, 1994.

Friedlander, A. H. \& Lande, A. Panoramic radiographic identification of carotid arterial plaques. Oral Surg. Oral Med. Oral Pathol., 52(1):102-4, 198.

Friedlander, A. H.; Manesh, F. \& Wasterlain, C. G. Prevalence of detectable carotid artery calcification on panoramic radiographs of recent stroke victims. Oral Surg. Oral Med. Oral Pathol., 77(6):669-73, 1994.
Grases, F.; Santiago, C.; Simonet, B. M. \& Costa-Bauza, A. Sialolithiasis: mechanism of calculi formation and etiologic factors. Clin. Chim. Acta, 334(1-2):131-6, 2003.

Griniatsos, J.; Damaskos, S.; Tsekouras, N.; Klonaris, C. \& Georgopoulos, S. Correlation of calcified carotid plaques detected by panoramic radiograph with risk factors for stroke development. Oral Surg. Oral Med. Oral Pathol. Oral Radiol. Endod., 108(4):600-3, 2009.

Jacome, A. \& Abdo, E. Radiographic aspects of soft tissue calcification in maxillofacial region. Odontol. Clín. Cient., 9(1):25-32, 2010.

Kamikawa, R. S.; Pereira, M. F.; Fernandes, A. \& Meurer, M. I. Study of the localization of radiopacities similar to calcified carotid atheroma by means of panoramic radiography. Oral Surg. Oral Med. Oral Pathol. Oral Radiol. Endod., 101(3):374-8, 2006.

Monsour, P. A.; Romaniuk, K. \& Hutchings, R. Soft tissue calcifications in the differential diagnosis of opacities superimposed over the mandible by dental panoramic radiography. Aust. Dent. J., 36(2):94-101, 1991.

Mupparapu, M. \& Kim, I. Calcified carotid artery atheroma and stroke: A systematic review. J. Am. Dent. Assoc., 138(4):483-92, 2007.

Ram, S.; Siar, C. H.; Ismail, S. M. \& Prepageran, N. Prepageran N. Pseudo bilateral tonsilloliths: a case report and review of the literature. Oral Surg. Oral Med. Oral Pathol. Oral Radiol. Endod., 98(1):110-4, 2004.

Rebolledo, C. M.; Carbonell, M. Z. \& Díaz, C. A. Sialolitos en conductos y glándulas salivales. Revisión de literarura. Av. Odontoestomatol., 25(6):311-7, 2009.

Reuter, J. \& Hausamen, J. E. Sialolithiasis der glandula submandibularis im Kindesalter. Klin. Pädiatr., 188:2858, 1976.

Romano-Sousa, C. M.; Krejci, L.; Martins, F. M.; GraciosaFilho, R. G.; Martins, M. F.; Guedes, V. N. \& FenyoPereira, M. Diagnostic agreement between panoramic radiographs and color doppler images of carotid atheroma. J. Appl. Oral Sci., 17(1):45-8, 2009.

Scolozzi, P.; Laurent, F.; Lombardi, T. \& Richter, M. Intraoral venous malformation presenting with multiple phleboliths. Oral Surg. Oral Med. Oral Pathol. Oral Radiol. Endod., 96(2):197-200, 2003.

Silvestre-Donat, F. J.; Pla-Mocholi, A.; Estelles-Ferriol, E. \& Martinez-Mihi, V. Giant tonsillolith: Report of a case. Med. Oral Patol. Oral Cir. Bucal, 10(3):239-42, 2005. 
GARAY, I. \& OLATE, S. Consideraciones actuales en el estudio imagenológico de las calcificaciones de tejidos blandos en zona de ángulo mandibular. Int. J. Odontostomat., 7(3):455-464, 2013.

Tanaka, T.; Morimoto, Y.; Ansai, T.; Okabe, S.; Yamada, K. Tacuchi, A.; Awano S, Kito S, Takata Y, Takehara T, Ohba T.. Can the presence of carotid artery calcification on panoramic radiographs predict the risk of vascular diseases among 80-year-olds? Oral Surg. Oral Med. Oral Pathol. Oral Radiol. Endod., 101(6):777-83, 2006.

Thakur, J. S.; Minhas, R. S.; Thakur, A.; Sharma, D. R. \& Mohindroo, N. K. Giant tonsillolith causing odynophagia in a child: a rare case report. Cases J., 1(1):50, 2008.

White, S. \& Pharoah, M. Radiología Oral: Principios e interpretación radiográfica. 4ª ed. Madrid, Harcourt, 2002. pp.552-65.

Zenk, J.; Constantinidis, J.; Kydles, S.; Hornung, J. \& Iro, H. Klinische und diagnostische Befunde bei der Sialolithiasis. HNO, 47(11):963-9, 1999.
Correspondencia:

Prof. Dr. Sergio Olate

Unidad de Cirugía Oral y Maxilofacial

Universidad de La Frontera

Claro Solar No 115

Temuco

CHILE

Tel: +56 (45) 2325000

Email: sergio.olate@ufrontera.cl

Received: 12-01-2013

Accepted: 18-10-2013 